\title{
The microbiome in autonomic medicine and other updates in recent autonomic research
}

\author{
Mitchell G. Miglis ${ }^{1}\left[\right.$. Srikanth Muppidi ${ }^{1}$
}

Received: 22 July 2019 / Accepted: 23 July 2019 / Published online: 30 July 2019

๑) Springer-Verlag GmbH Germany, part of Springer Nature 2019

Keywords Microbiome $\cdot$ Hypertension $\cdot$ Multiple system atrophy $\cdot$ Synuclein $\cdot$ Consensus criteria $\cdot$ Epigallocatechin

\section{The microbiome in autonomic medicine}

The term "microbiome" was relatively unknown 10 years ago, yet today it is a part of everyday conversation. This popular shift reflects an explosion of scientific literature on the role of symbiotic microorganisms in our bodies, health, and disease. The enteric nervous system (ENS) consists of an estimated 100 million neurons. That is one-thousandth the number of neurons in the brain, and about one-tenth the number of neurons in the spinal cord. Most of these neurons regulate autonomic functions. In addition, there is estimated to be at least one colonized cell living within us for every human cell in our body. The role of the microbiome in medicine is a fascinating subject, and we are only scratching the surface.

While there have been several animal models that have demonstrated the influence of gut microbiota and cardiovascular disease, Sun and colleagues have just recently reported on the first large-scale study in humans. Published in the May 2019 edition of Hypertension [1], the gut microbiota composition and blood pressure substudy of the CARDIA (Coronary Artery Risk Development in Young Adults) study enrolled 529 socioeconomically and racially diverse middle-aged adults from 4 US centers (Birmingham, AL; Chicago, IL; Minneapolis, MN; Oakland, CA) and has followed them for over 30 years. The CARDIA project was started in 1985 as a multicenter longitudinal cohort to help determine how genetic and lifestyle factors influence cardiovascular risk over time. Sun and colleagues analyzed stool samples from 529 participants at their 30-year follow-up

Mitchell G. Miglis

mmiglis@stanford.edu

1 Stanford Medical Center, Stanford Neurosciences Health Center, 213 Quarry Road 2nd Floor, Palo Alto, CA 94304, USA visit and sequenced these samples for the $16 \mathrm{~S}$ ribosomal RNA (rRNA) prokaryotic marker gene, a gene often used in phylogenetic studies due to the fact that it is highly conserved between different species of bacteria. This allowed the researchers to then determine the genera of microbiota present in the GI tracts of participants.

What the authors found was both fascinating and not surprising: the more diverse a subject's microbial diversity, the less likely they were to have hypertension. In other words, measures of within-person microbial diversity were inversely associated with hypertension. These differences remained significant even after adjusting for demographic and health behavior covariates. There were several types of bacteria that seemed to offer a protective benefit, such as Sporobacter and Anaerovorax bacteria that commonly produce short-chain fatty acids (SCFAs), which can lower blood pressure. When body mass index and waist circumference were controlled for, however, the association between the presence of these particular bacteria and hypertension failed to reach statistical significance.

The most notable limitation of this study is the inability to prove causality: was the microbiota diversity cause or effect? Animal studies have demonstrated evidence of a causal relationship, including the action of specific shortchain fatty acids derived from gut microbes that modulate G-protein-coupled receptors to regulate blood pressure [2], among other biochemical and immune-mediated mechanisms. However, human data are lacking. Another limitation from the autonomic perspective is the exclusion of individuals with recent vomiting or constipation, both of which are common in autonomic disorders. Nonetheless, this study represents the first of its kind to examine the relationship between microbiota and blood pressure in a large human cohort, and stimulates thoughts on the coming era of personalized medicine, where stool sampling might become just as 
common as blood sampling in diagnosing disease and optimizing health. We are closer to this reality than we realize.

\section{Multiple system atrophy: another negative trial and the need for accurate diagnosis}

A diagnosis of multiple system atrophy (MSA), the most aggressive $\alpha$-synucleinopathy, implies a relentless course of progressive autonomic and motor impairment, with no available disease-modifying treatment. Early and accurate diagnosis remains a critical step for drug development, and we are still in the process of developing clinical tools to improve diagnosis. The diagnosis of MSA is based on the second consensus statement [3], which classifies MSA as "definitive" based on neuropathological confirmation, and "possible" or "probable" based on clinical criteria. Additional unique clinical features ("red flags") help to improve diagnostic accuracy. A recent online publication by Miki et al. in the journal Brain titled "Improving diagnostic accuracy of multiple system atrophy: a clinicopathological study" highlights the limitations of this methodology [4]. The authors retrospectively reviewed 203 cases with a clinical diagnosis of MSA from their center, of whom $160(78.8 \%)$ had confirmed MSA based on post-mortem neuropathological analysis. Other patients had alternative diagnoses, the most common being Lewy body dementia (DLB) or progressive supranuclear palsy (PSP). Further multiple logistical regression analyses revealed an increased likelihood of MSA diagnosis compared to DLB if patients had either orthostatic hypotension or bladder dysfunction requiring urinary catheterization (OR 2.0, $p=0.021)$. Onset of autonomic dysfunction within 3 years of disease onset was also useful in differentiating MSA from PSP (OR 3.4, $P=0.023)$. MSA patients had a higher number of "red flag" clinical signs compared to other diseases. Improved usage of these parameters in clinical practice is likely to improve overall diagnostic accuracy, and a task force to amend the current consensus criteria sponsored by the Movement Disorders Society is already in place [6].

In the meantime, another negative MSA treatment trial has been published, which speaks to the need for greater accuracy of diagnosis. The polyphenol epigallocatechin gallate, a component of green tea extract and a dietary supplement approved in many countries, had shown promising results in animal models of synucleinopathy. This prompted a multicenter, double blind, randomized clinical trial to study the efficacy of this compound in patients with MSA. The results were recently published by Levin et al. in Lancet Neurology [5]. In their paper titled "Safety and efficacy of epigallocatechin gallate in multiple system atrophy (PROMESA): a randomized, double-blind, placebo-controlled 52 week trial," the authors recruited 92 patients randomly assigned to epigallocatechin gallate or placebo. Of these patients, 67 completed treatment and 64 completed the study. The primary endpoint was change in the motor score of the Unified Multiple System Atrophy Rating Scale (UMSARS). Safety analysis was also performed. There was no difference in the mean change in UMSARS score from baseline to week 52 between the epigallocatechin gallate (5.66 [SE 1.01]) and placebo (6.60 [0.99]) groups (mean difference -0.94 [SE $1.41 ; 95 \%$ CI -3.71 to 1.83 ]; $p=0.51$ ). During the study period, four patients in the epigallocatechin gallate group and two in the placebo group died. Two patients in the epigallocatechin gallate group had to stop treatment due to hepatotoxicity. Interestingly, additional analysis to evaluate for MRI evidence of disease progression revealed that patients in the epigallocatechin gallate group had a slower rate of striatal atrophy compared to the placebo group. To complicate matters further, patients who participated in the MRI analysis were enrolled at high-recruitment centers, and sensitivity analysis revealed that there were group differences between patients recruited at high-recruitment centers and patients recruited from other centers. This raises the interesting question of diagnostic accuracy, and if experienced recruitment centers are better equipped to recruit a more uniform group of patients with a higher diagnostic accuracy rate. This study highlights the urgent need for better MSA treatments, and the fact that better options for better treatment always begin with proper diagnosis.

Funding None

\section{Compliance with ethical standards}

Conflict of interest The authors declare that they have no competing interests.

\section{References}

1. Sun S, Lulla A, Sioda M et al (2019) Gut microbiota composition and blood pressure. Hypertension 73(5):998-1006

2. Pluznick JL, Protzko RJ, Gevorgyan H et al (2013) Olfactory receptor responding to gut microbiota-derived signals plays a role in renin secretion and blood pressure regulation. Proc Natl Acad Sci USA 110(11):4410-4415

3. Gilman S, Wenning GK, Low PA et al (2008) Second consensus statement on the diagnosis of multiple system atrophy. Neurology 71(9):670-676

4. Miki Y, Foti SC, Asi YT et al (2019) Improving diagnostic accuracy of multiple system atrophy: a clinicopathological study. Brain. https ://doi.org/10.1093/brain/awz189

5. Levin J, Maaß S, Schuberth M et al (2019) Safety and efficacy of epigallocatechin gallate in multiple system atrophy (PROMESA): a randomised, double-blind, placebo-controlled trial. Lancet Neurol 18(8):724-735

6. Stankovic I, Quinn N, Vignatelli L et al (2019) A critique of the second consensus criteria for multiple system atrophy. Mov Disord 34(7):975-984 